Revista de Psicología Vol. 32 (2), 2014 (ISSN 0254-9247)

\title{
Validación de la escala de cinismo organizacional: un estudio con trabajadores argentinos
}

\author{
Solana Salessi ${ }^{1}$ y Alicia Omar ${ }^{2}$ \\ Pontificia Universidad Católica Argentina, Universidad Nacional de Rosario \\ Consejo Nacional de Investigaciones Cientificas y Técnicas (CONICET)
}

\begin{abstract}
El objetivo del estudio consistió en validar la Escala de Cinismo Organizacional de Brandes para su empleo con trabajadores argentinos. Se analizó la equivalencia funcional del instrumento. Se administró una versión adaptada a una muestra de 396 empleados, quienes respondieron también una selección de instrumentos para explorar confianza en la organización, compromiso organizacional y satisfacción laboral. El análisis factorial exploratorio mostró una estructura de tres factores que explicaron el $68.8 \%$ de la varianza total. El análisis factorial confirmatorio condujo a re-especificar el modelo, eliminando tres ítems. La validez de constructo quedó demostrada en base a los análisis de correlación efectuados. La confiabilidad del instrumento alcanzó niveles satisfactorios. Se puntualizan las debilidades y fortalezas de la investigación realizada. Se sugiere una agenda para futuros estudios.

Palabras clave: cinismo, organizaciones, trabajadores, validez
\end{abstract}

\section{Validation of the organizational cynicism scale: A study with Argentinean workers}

The aim of this study was to validate the Brandes's Organizational Cynicism Scale in order to use it with Argentinean workers. Functional equivalence of the instrument was analyzed. The adapted version was administered to a sample of 396 workers; who also answered a selection of instruments to explore organizational trust, organizational commitment and job satisfaction. Exploratory factor analysis showed a three-factor structure that explained $68.8 \%$ of the total variance. Confirmatory factor analysis led to re-specification of the model,

1 El presente estudio fue realizado en el marco de la beca doctoral otorgada por el CONICET a la primera autora, dirigida por la segunda autora.

Licenciada en Psicología y becaria doctoral del CONICET. Profesora asistente, Cátedra de Psicología, Profesorado Superior. Facultad de Derecho y Ciencias Sociales del Rosario, Pontificia Universidad Católica Argentina. Dirección Postal: Balcarce 855, $9^{\circ} \mathrm{A}$ (S2000DNQ) Rosario, Argentina. Contacto: solanasalessi@gmail.com

2 Doctora en Psicología, Investigadora científica del CONICET, Instituto de Investigaciones, Facultad de Humanidades y Artes, Universidad Nacional de Rosario. Docente categoría 1 de Carreras de Postgrado. Dirección Postal: Italia 1365, 1º A, (S2000DFA) Rosario, Argentina. Contacto: agraomar@yahoo.com 
eliminating three items. Construct validity was demonstrated on the basis of correlation analysis. Instrument reliability reached satisfactory levels. Weaknesses and strengths of the research are pointed out. A new agenda for future research is suggested.

Key words: cynicism; organizations; employees; statistical validity 
El estudio del cinismo se ha posicionado como una temática atractiva para los especialistas en comportamiento organizacional durante las últimas dos décadas, aunque las primeras investigaciones sobre el tema se realizaron en el ámbito de la psicología de la personalidad a mediados del siglo pasado (Cook \& Medley, 1954; Sarnoff, 1960). El interés por el cinismo, como respuesta de los trabajadores frente a las nuevas realidades laborales, fue propiciado por las transformaciones socio-político-económicas que atravesaron las postrimerías del siglo XX y los albores del siglo XXI (Kanter \& Mirvis, 1989). Aunque se le reconoce a Brooks y Vance (citados en Brandes \& Das, 2006) el mérito de haber utilizado por primera vez el término cinismo organizacional (CO), fue a partir de la publicación de Dean, Brandes y Dharwadkar (1998) que adquirió la relevancia que posee en la actualidad.

Inicialmente, el $\mathrm{CO}$ fue conceptualizado como una creencia de que el cambio organizacional es posible pero improbable, debido a la ineptitud o falta de voluntad de los responsables de concretarlo (Watt \& Piotrowski, 2008). En la literatura contemporánea, el constructo es definido como una actitud laboral negativa que los empleados desarrollan hacia la organización en la que trabajan (Brandes, Das \& Hadani, 2006). Desde la perspectiva de Dean y colaboradores (1998), el CO es un conjunto de pensamientos pesimistas, afectos negativos y comportamientos críticos dirigidos hacia la organización en su conjunto o hacia diversos elementos del ambiente laboral (por ejemplo, las políticas, los valores, las prácticas y las personas). Por lo que constituye un constructo complejo, que puede subsumir otras formas de cinismo frecuentemente estudiadas como variantes específicas e independientes. Tal es el caso, por ejemplo, del cinismo hacia el cambio en general (Reichers, Wanous \& Austin, 1997), el cinismo hacia las corporaciones (Bateman, Sakano \& Fujita, 1992), y el cinismo hacia la alta gerencia (Andersson \& Bateman, 1997). 
En la actualidad, el CO es considerado como un constructo multidimensional, conformado por ideas, afectos y tendencias a la acción. El concepto se enraizó en la convicción que los principios de honestidad y justicia han sido quebrantados en favor de los intereses personales de la alta gerencia. Los cínicos organizacionales creen firmemente que las organizaciones en las que trabajan son injustas y egoístas. Consideran que se encuentran impedidos de participar en los procesos de toma de decisiones, y que existen motivaciones ocultas subyacentes a las informaciones que se les comunican explícitamente (Qian \& Daniels, 2008). Sienten suspicacia y recelo frente a las promesas de cambio y mejora; y el incumplimiento de las mismas les genera desilusión, enojo y sentimientos de traición (Salessi, 2011). Asimismo, se ha señalado (Dean, Brandes \& Dharwadkar, 1998) que el empleado cínico puede sentirse moralmente superior y experimentar una secreta complacencia por ello. Las manifestaciones observables del CO abarcan desde declaraciones explícitas sobre la falta de principios y de ética, hasta conductas claramente contraproducentes para la organización (Chiaburu, Peng, Oh, Banks \& Lomeli, 2013). Entre los cínicos organizacionales son frecuentes las miradas de complicidad y las sonrisas sarcásticas, así como el humor cínico y la crítica destructiva (Brandes \& Das, 2006). El humor cínico contribuye a sobrellevar los sentimientos negativos propios del CO y, por lo general, se expresa bajo la forma de ridiculización de las insignias organizacionales. De este modo, las burlas y bromas promueven una sensación temporal de alivio frente a las presiones organizacionales (Fleming \& Spincer, 2003). La crítica destructiva, por el contrario, impulsa el malestar general y socava las relaciones con los compañeros de trabajo, operando como catalizador de una espiral de negatividad ascendente. Constituye, en última instancia, un modo de expresar la impotencia, la desesperanza y el pesimismo inherentes al cinismo, y actúa como un refuerzo positivo que sostiene dicha actitud en el tiempo (Kanter \& Mirvis, 1989).

El CO ha demostrado ser un fenómeno altamente perjudicial para las organizaciones. Entre sus consecuencias negativas se destacan la menor productividad (Neves, 2012), la resistencia al cambio (Barton 
\& Ambrosini, 2013), y la falta de compromiso con la organización y el trabajo (Türköz, Polat \& Coţar, 2013). Los cínicos organizacionales no se sienten obligados a realizar comportamientos que sobrepasen lo estrictamente prescripto para su puesto, por lo que se involucran escasamente en conductas de ciudadanía organizacional (Evans, Goodman \& Davis, 2011). Sin embargo, los efectos nocivos del CO no se agotan en la esfera laboral. Recientemente se ha comunicado que el mantenimiento de esta actitud en el tiempo también conlleva efectos perjudiciales para los empleados (Salessi \& Omar, 2014). En este sentido, los cínicos organizacionales presentan menores niveles de satisfacción y bienestar, junto a una elevada predisposición a experimentar estrés y burnout. A su vez, la desconfianza y la cautela propias de las actitudes cínicas pueden tener efectos inhibidores y perturbadores en las relaciones interpersonales erosionando, incluso, las redes de apoyo social del cínico.

Por sus implicancias sobre la eficacia de las organizaciones y sobre la salud mental de los trabajadores, el estudio del $\mathrm{CO}$ ha trascendido los contextos organizacionales norteamericanos en los cuales emergió (Dean et al., 1998). En este sentido, se registran esfuerzos para estudiar sus antecedentes y consecuencias tanto en países asiáticos y europeos (Grama, 2013; Gkorezis, Petridou \& Xanthiakos, 2014; Kan, 2014), como latinoamericanos (Didier Pino, Martí del Campo \& Valdenegro Ibarra, 2012; Martínez Lugo et al., 2005; Salessi, 2011; Salessi \& Omar, 2014).

En paralelo a los trabajos tendientes a establecer las bases conceptuales del CO y diferenciarlo de otros constructos similares, se han llevado a cabo intentos sistemáticos por operacionalizarlo. La revisión de la literatura especializada muestra que en la medición del CO se han privilegiado las escalas auto descriptivas frente a otras alternativas de recolección de datos (por ejemplo, observaciones o entrevistas). Entre las diferentes propuestas para medir el cinismo en las organizaciones, la escala elaborada por Brandes, Dharwadkar y Dean (1999) es uno de los instrumentos más utilizados en las investigaciones actuales. Su popularidad se basa en su capacidad para explorar en detalle cada una 
de las dimensiones que integran el constructo, a diferencia de otras escalas de $\mathrm{CO}$ que solo miden aspectos parciales o variantes específicas del fenómeno (Stanley, Meyer \& Topolnytsky, 2005; Wanous, Reichers $\&$ Austin, 2000).

La validación inicial de la escala fue desarrollada por Brandes et al. (1999) con una muestra de 393 trabajadores de una industria norteamericana, de los cuales 264 eran empleados rasos y 129 se desempeñaban como supervisores. La matriz de datos permitió extraer tres factores que explicaron el $54.78 \%$ de la varianza total del constructo. El primer factor, fue denominado "Cinismo Organizacional Cognitivo" (5 ítems; $\alpha=.86$ ), ya que los ítems que lo integran exploran la percepción de inconsistencia entre las políticas y las prácticas organizacionales. El segundo factor, etiquetado como "Cinismo Organizacional Afectivo" ( 4 ítems; $\alpha=.80$ ), evalúa las emociones y sentimientos negativos hacia la organización empleadora. El tercer factor, identificado como "Cinismo Organizacional Comportamental" ( 4 ítems; $\alpha=.78$ ), explora las conductas de críticas, quejas y sarcasmo respecto a la organización y su funcionamiento. Los 13 reactivos que conforman el instrumento original, adoptan el formato de oraciones afirmativas frente a las cuales los sujetos deben indicar la frecuencia de su ocurrencia sobre una escala Likert de 5 puntos. El instrumento proporciona un puntaje global en $\mathrm{CO}$, así como puntajes parciales correspondientes a cada una de las tres dimensiones que lo integran. Hasta el momento, se han concretado algunos pocos estudios de validación de esta escala, ya sea para su empleo en contextos europeos (Bobbio, Manganelli Rattazzi \& Spadaro, 2006) o asiáticos (Karacaoğlu e İnce, 2012). Sin embargo, no se dispone de una versión traducida, adaptada y validada para su empleo con poblaciones hispanoparlantes. Por lo que en un intento por llenar este vacío empírico-instrumental, el objetivo del presente trabajo consistió en la adaptación y validación de la escala de CO de Brandes y colaboradores (1999) para su empleo en el ámbito argentino. 


\section{Método}

De las diferentes formas de adaptación, se optó por el método propuesto por Herdman, Fox-Rushby y Badía (1998), popularizado por Hasselmann y Reichenheim (2003). Este método permite establecer la equivalencia funcional entre el instrumento foráneo y su versión adaptada, y consta de un conjunto de pasos a seguir: equivalencia conceptual, semántica, operacional y de medición.

La equivalencia conceptual se refiere a la equivalencia de cada concepto entre la cultura donde fue desarrollado el instrumento y la cultura objetivo (en la que se aplicará el instrumento). Con tal propósito se efectuó una exhaustiva revisión bibliográfica sobre el modo en que el concepto "cinismo organizacional" es entendido en la cultura argentina. El análisis y discusión de los hallazgos permitió concluir que la naturaleza del concepto era similar tanto para la cultura de origen (norteamericana) como para la cultura objetivo (argentina). Como parte de este proceso, todos los ítems de la escala original fueron sometidos a una intensa revisión crítica a cargo de expertos. La evaluación de los especialistas determinó que el espectro explorado por el instrumento cubría los tres dominios del constructo identificados por Brandes y colaboradores (1999) y que los ítems eran pertinentes a la población, demandando tan solo mínimas modificaciones de traducción que de ningún modo alterarían su sentido original.

La equivalencia semántica consiste en la traducción del instrumento conservando el significado entre idiomas diferentes. En este caso el análisis de la equivalencia semántica fue realizado en cuatro etapas: a) el instrumento original fue traducido del inglés al español (argentino); b) expertos en inglés retradujeron la versión española al inglés; c) traductores de inglés compararon las dos formas del instrumento 'a ciegas' a efectos de identificar la concordancia entre el ítem original y el traducido; d) tomando en cuenta las apreciaciones de los especialistas los autores del presente trabajo discutieron y decidieron la inclusión de los ítems que integrarían la versión argentina del instrumento. Esta versión prototípica fue administrada a una muestra no probabilística de la 
población objetivo, integrada por 86 estudiantes de posgrado; quienes, a su vez, trabajaban en diferentes organizaciones radicadas en la ciudad de Rosario y alrededores. El 56\% de la muestra estaba conformada por varones. La edad promedio de los participantes fue de 32.81 años $(D E=5.64)$, en tanto que la antigüedad laboral media fue de 3.74 años $(D E=2.24)$.

La finalidad de este estudio preliminar fue explorar la equivalencia operacional (mantenimiento de las características operativas del universo original mediante el empleo de ciertas normas antes y durante la aplicación de los instrumentos), básicamente en lo referente a tiempo para completar la escala, claridad de las instrucciones para efectuar la tarea y adecuación semántica y sintáctica de los ítems. Este estudio demostró que los ítems eran bien comprendidos, que las instrucciones estaban claramente indicadas y que la escala elegida para responder la escala (Likert de 5 puntos) no generaba dificultades.

La equivalencia de medición tiene como propósito analizar las propiedades psicométricas de un instrumento a través del cálculo de medidas de confiabilidad y validez. En este caso, se analizó la validez factorial exploratoria (AFE) mediante un análisis de componentes principales con rotación oblimix. A continuación, se realizó un análisis factorial confirmatorio (AFC) a efectos de verificar la adecuación del modelo indicado por el AFE. La validez de constructo se determinó a través de la correlación con otros conceptos que, a partir de la revisión de la literatura especializada, surgían como relevantes con respecto al CO. Se calculó la confiabilidad mediante el cómputo de coeficientes de consistencia interna (alpha de Cronbach). Para el estudio de la equivalencia de medición se empleó la muestra y los instrumentos que se describen a continuación:

\section{Participantes}

Se trabajó con una muestra no probabilística integrada por 396 trabajadores argentinos (53\% varones), pertenecientes a distintas organizaciones públicas y privadas radicas en la zona centro de Argentina. 
La edad de la muestra osciló entre 21 y 69 años, encontrándose el promedio en torno a los 32.42 años $(D E=9.48)$. La antigüedad laboral media fue de 7.73 años $(D E=8.50)$. El $43 \%$ de los encuestados tenía formación superior completa (universitaria o terciaria), en tanto que el resto había concluido sus estudios secundarios. El 82.2\% se desempeñaba en relación de dependencia. El 37.5\% ocupaba mandos medios-altos, siendo jefes, supervisores o gerentes de área respectivamente. El 64\% de los participantes trabajaba en organizaciones de gestión privada. En cuanto a los ramos de actividad, el 37.2\% de la muestra se desempeñaba en el sector servicios, el $33.9 \%$ en la industria, el $18.6 \%$ en educación y el $10.3 \%$ restante en el ámbito de la salud.

\section{Instrumentos}

Los participantes respondieron un cuadernillo integrado por la versión adaptada de la Escala de CO (13 ítems con formato de respuesta tipo Likert de 5 puntos, variando de 1 = nunca a 5 = siempre), y una selección de instrumentos estandarizados, desarrollados para medir los constructos que se detallan a continuación.

Confianza organizacional: fue medida mediante la adaptación argentina (Flores, 2011) del Inventario de Confianza Organizacional (Oliveira \& Tamayo, 2008). La adaptación incluye 20 ítems distribuidos en cinco subescalas rotuladas como: Promoción del crecimiento del empleado ( 4 ítems; $\alpha=.84$, ej.: "Se ofrecen condiciones reales para que el empleado se desarrolle"); Solidez organizacional (5 ítems; $\alpha=.85$; ej.: "Es conocida por su poder económico"); Reconocimiento financiero organizacional ( 4 ítems; $\alpha=.71$; ej.: "El trabajo del empleado es reconocido a través del salario"); Normas relativas al despido de los empleados (3 ítems; $\alpha=.83$; ej.: "Los empleados son despedidos en cualquier momento, independientemente de las normas legales"); Patrones éti$\cos$ ( 4 ítems; $\alpha=.84$; ej.: "Ser honesto con los clientes es su principio ético"). Cada ítem es valorado sobre una escala tipo Likert de cinco puntos, que va desde 1 (totalmente en desacuerdo) hasta 5 (totalmente de acuerdo). 
Compromiso organizacional: fue explorado a través de la adaptación argentina (Omar, 2006) de la Escala de Compromiso Organizacional de Meyer y Allen (1991). Dicho instrumento se encuentra conformado por 18 ítems distribuidos equitativamente en tres factores, a saber: Compromiso afectivo ( $\alpha=.87$; ej.: "Yo estaría feliz si pasara el resto de mi carrera en la empresa donde trabajo"); Compromiso calculativo ( $\alpha=.82$; ej.: "En la actualidad permanezco en mi empresa tanto por necesidad como por deseo"); y Compromiso normativo ( $\alpha=.69$; ej.: "Yo no siento ninguna obligación para permanecer en mi actual empleo"). Cada ítem es valorado sobre una escala Likert de cinco puntos, que va desde 1 (totalmente en desacuerdo) hasta 5 (totalmente de acuerdo).

Satisfacción laboral: fue evaluada mediante la Escala de Satisfacción Laboral Genérica de Mac Donald y Mac Intyre (1997), adaptada por Omar (2011). El instrumento cuenta con adecuadas propiedades psicométricas y es aplicable a los más diversos ámbitos ocupacionales. Está integrado por siete ítems $(\alpha=.79$; ej.:"Mi trabajo me permite desarrollar todo mi potencial"), y proporciona una estimación global del grado de satisfacción laboral. Cada ítem es valorado sobre una escala tipo Likert de cinco puntos, variando desde 1 (totalmente en desacuerdo) a 5 (totalmente de acuerdo).

El protocolo de exploración incluyó también una hoja de consentimiento informado, $y$ un apartado diseñado para recabar información acerca de la edad, el género, el nivel de escolaridad y antigüedad laboral de los participantes; así como sobre el puesto que desempeñaban (jefe/ supervisor/gerente o empleado), el ramo de actividad de su organización (servicios, industria, educación y salud) y el tipo de gestión de la misma (pública o privada).

\section{Procedimiento}

En primera instancia, se tomó contacto con diversas organizaciones públicas y privadas localizadas en ciudades ubicadas en la zona central del país, invitándolas a participar del estudio. Con aquellas que aceptaron colaborar se pautaron días y horarios para concretar la 
recolección de los datos, procurando que el proceso se realizara en el seno de las mismas instituciones, y en los horarios y lugares designados por cada organización para tal efecto. Tras explicar el propósito del estudio, la modalidad de respuesta, asegurar el anonimato de todos los participantes y la confidencialidad de los datos recabados, se procedió a seleccionar solo a aquellos empleados que habían aceptado participar voluntariamente. Los sujetos recibieron instrucciones sobre la mecánica de respuesta. Se los invitó a completar los cuestionarios de manera individual o en pequeños grupos. Las dudas que surgieron fueron aclaradas individualmente y en forma personalizada durante la instancia de administración.

\section{Resultados}

El grado de equivalencia semántica se determinó en función de dos categorías de análisis. Por un lado, el significado referencial, vinculado con la concordancia en términos de traducción literal entre el ítem original y el ítem traducido. Por otro lado, el significado general, correspondiente a la articulación de ideas entre el ítem original y su re-traducción. El primero fue evaluado sobre una escala visual analógica en la que la equivalencia entre pares fue juzgada de 0 a $100 \%$. El segundo fue evaluado por dos traductores en función de cuatro niveles de equivalencia: a) inalterado; b) poco alterado; c) bastante alterado; y d) completamente alterado. Ambos profesionales acordaron que la adaptación semántica de la escala CO mostraba adecuados niveles de concordancia traducción-retraducción. Los resultados de este último análisis se presentan en la Tabla 1 . 


\section{Tabla 1}

Niveles de equivalencia semántica entre el item originaly su segunda traducción

\begin{tabular}{lcc}
\hline \multirow{2}{*}{ Nivel de equivalencia } & \multicolumn{2}{c}{$\begin{array}{c}\text { Traducción-segunda traducción } \\
\text { (inglés-español-inglés) }\end{array}$} \\
\cline { 2 - 3 } & Traductor 1 & Traductor 2 \\
\hline Inalterado & $11 / 13=84 \%$ & $10 / 13=77 \%$ \\
\hline Poco alterado & $2 / 13=16 \%$ & $3 / 13=23 \%$ \\
\hline Bastante alterado & $0 / 13=0 \%$ & $0 / 13=0 \%$ \\
\hline Completamente alterado & $0 / 13=0 \%$ & $0 / 13=0 \%$ \\
\hline
\end{tabular}

En lo que hace a la equivalencia de medición, en primera instancia se llevó a cabo un AFE, a través del método de componentes principales con rotación Oblimim, ya que se consideraba la hipótesis de que los factores de la escala estuvieran correlacionados. La matriz de datos fue considerada factorizable habida cuenta que el test de esfericidad de Bartlett fue significativo $\left(\chi^{2}=5296.92 ; p<.001\right)$, y el índice de adecuación muestral de Kaiser-Meyer-Olkin arrojó un valor de .92. Tal como lo indicó el gráfico de sedimentación (scree plot), se extrajeron tres factores que explicaron el $68.68 \%$ de la varianza total del constructo. Para la asignación de los ítems a cada factor se utilizaron dos criterios: (a) que el reactivo estuviera conceptualmente relacionado con el factor considerado, y (b) que tuviera un peso factorial igual o mayor a .40 en el factor correspondiente (Tabachnick \& Fidell, 2007).

El análisis de los ítems que saturaron sobre cada factor llevó a rotular al factor 1 como "Ideas cínicas", ya que aglutinó 5 ítems referidos a la percepción de incoherencia e inconsistencia entre las políticas y prácticas organizaciones. El factor 2 fue identificado como "Conductas cínicas”, ya que los 4 ítems que pesaron sobre este, están referidos a la presencia de conductas críticas, quejumbrosas y sarcásticas. Por último, el factor 3 fue rotulado como "Emociones cínicas", ya que los 4 ítems que lo integran están vinculados con la afectividad negativa. 
La consistencia interna (alpha de Cronbach) correspondiente a cada factor fue superior a .70 indicando una aceptable confiabilidad (Nunnally \& Bernstein, 1994). La Tabla 2 presenta la composición de la escala, las cargas factoriales correspondientes a cada ítem, la varianza explicada y los coeficientes $(\alpha)$ de los factores.

\section{Tabla 2}

Composición, carga factorial, varianza explicada y coeficientes $\alpha$ de los factores correspondientes a la versión adaptada de la Escala $C O$

$\mathrm{N}^{\circ}$ Contenido del ítem

Factor 1 Factor 2 Factor 3

Ideas cínicas

1 Creo que mi jefe dice una cosa y hace $\quad .90$ otra

2* Las políticas, objetivos y prácticas de mi empresa parecen tener poco en común

3 Cuando mi jefe dice que va a hacer algo, $\quad .82$ dudo si realmente lo hará

$4^{*}$ En mi empresa se pide una cosa a los empleados, pero se recompensa otra

5 Encuentro poca relación entre lo que mi .85 jefe dice que hará y lo que realmente hace

Conductas cínicas

6 Me quejo con mis amigos de las cosas que .88 suceden en mi empresa

7 Puedo intercambiar miradas de .79 complicidad con mis compañeros de trabajo

8 Comento con otras personas cómo andan las cosas en mi empresa

9 Critico las prácticas y políticas de mi empresa con los demás. 


\section{Emociones cínicas}

10 Cuando pienso en mi empresa me siento .73 enojado

11 Cuando pienso en mi empresa me siento tenso

$12^{*}$ Cuando pienso en mi empresa me siento ansioso

13 Cuando pienso en mi empresa me siento molesto

\begin{tabular}{lccc}
\hline Porcentaje de varianza explicada & $36.52 \%$ & $21.28 \%$ & $11.09 \%$ \\
\hline Coeficiente alpha de Cronbach $(\alpha)$ & .91 & .96 & .86 \\
\hline
\end{tabular}

Nota. *ítems eliminados en el segundo AFC; por lo que no forman parte de la versión final de la escala.

Seguidamente, se llevó a cabo un AFC con miras a poner a prueba el modelo de tres factores correlacionados y 13 variables observables indicado por el AFE (Tabla 2). Para dicho análisis se empleó el método de estimación de la máxima verosimilitud y se calcularon diversos índices de ajuste, siguiendo las sugerencias de Byrne (2010). En este sentido, se optó por la combinación que reúne los índices $\chi^{2}, \chi^{2}$ relativo (CMIN/gl), índice de bondad de ajuste (GFI) y su variante ajustada (AGFI), índice de ajuste comparativo (CFI), y error de aproximación de la raíz cuadrada media (RMSEA). Lo esperable es que la prueba $\chi^{2}$ no arroje valores significativos al nivel del 5\%; que el índice CMIN/gl arroje valores menores a 3; que los índices GFI, AGFI y CFI alcancen un valor de .90 o superior; y que el índice RMSEA muestre valores por debajo de .08 (Schumacker \& Lomax, 2004).

Los resultados obtenidos con el primer modelo probado (Modelo A, Tabla 3) no fueron plenamente satisfactorios. Si bien los índices de bondad de ajuste (GFI) y de ajuste comparativo (CFI) mostraron valores adecuados; el índice $\chi^{2}$ fue significativo y la razón del $\chi^{2}$ sobre los grados de libertad (CMIN/gl) arrojó valores superiores a los esperados. 
Sumado a eso, el índice RMSEA fue mayor a lo sugerido. Tales indicadores demostraban que la estructura del modelo teórico propuesto era significativamente diferente a la indicada por la matriz de covarianza de los datos. Por lo tanto, se procedió a re-especificar el modelo examinando previamente las cargas factoriales, los índices de modificación y la matriz residual de la matriz de las predicciones de la covarianza y correlación (Hair, Black, Babin, Anderson \& Tatham, 2006). En función de los resultados derivados de dicho examen, se decidió descartar tres ítems y volver a calcular el ajuste del modelo. Eliminar ítems para mejorar la estructura factorial de un instrumento constituye un recurso legítimo, habida cuenta que conserva la estructura general del modelo original, pero únicamente con los indicadores más convenientes (Kline, 2011). En este sentido, fueron eliminados los siguientes reactivos: ítem 2 ("Las políticas, objetivos y prácticas de mi empresa parecen tener poco en común"); ítem 4 ("En mi empresa se pide una cosa a los empleados, pero se recompensa otra"); e ítem 12 ("Cuando pienso en mi empresa me siento ansioso"). A continuación, se procedió a efectuar un segundo AFC, probando ahora un modelo conformado por 3 factores correlacionados y 10 variables observables (Modelo B, Tabla 3).

\section{Tabla 3}

Indices de bondad de ajuste de los modelos de CO examinador

\begin{tabular}{lcccccccc}
\hline Modelo & $\chi^{2}$ & $d f$ & $P$ & CMIN/gl & GFI & AGFI & CFI & RMSEA \\
\hline Modelo A & 283.01 & 62 & .00 & 4.56 & .91 & .86 & .96 & .09 \\
Modelo B & 50.16 & 32 & .22 & 1.57 & .98 & .96 & .99 & .03 \\
\hline
\end{tabular}

Nota $. \chi^{2}=$ chi-cuadrado; $g l=$ grados de libertad; $\mathrm{CMIN} / \mathrm{gl}=\chi^{2}$ relativo; GFI $=$ índice de bondad de ajuste; AGFI = variante ajustada del GFI; CFI = índice de ajuste comparativo; RMSEA = error de aproximación de la raíz cuadrada media.

Tal como puede observarse en la Tabla 3, el Modelo B mostró un ajuste mejor en comparación al Modelo A; razón por la cual, se optó por conservarlo como definitivo. La estructura final de la versión adaptada de la escala de CO se muestra en la Figura 1. 


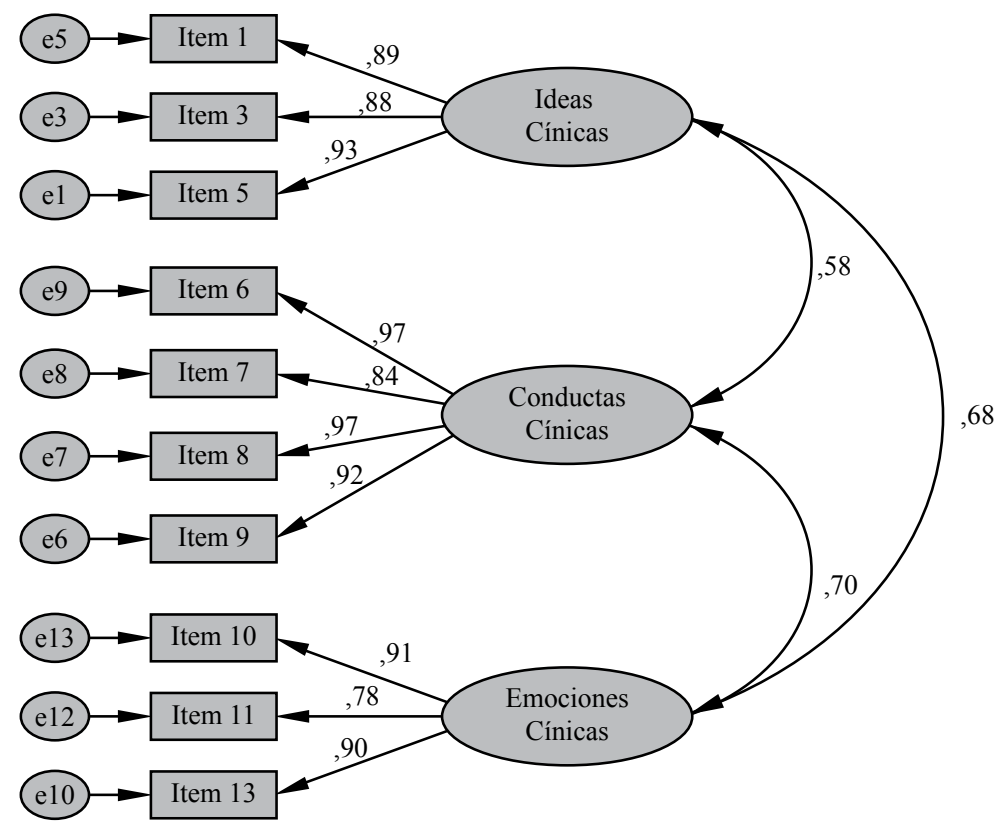

Figura 1. Modelo de CO re-especificado

Paralelamente, se procedió a estimar nuevamente la confiabilidad del instrumento, (integrado ahora por 10 ítems), a través del cálculo de los respectivos coeficientes alpha de Cronbach. Se obtuvieron valores adecuados de consistencia interna para las tres dimensiones del CO (Ideas Cinicas $\alpha=.93$; Conductas Cínicas $\alpha=.96$; Emociones Cinicas $\alpha=.89)$, así como para la escala total ( $\mathrm{CO} \alpha=.93)$.

Finalmente, la validez de constructo fue explorada mediante el cómputo de un conjunto de coeficientes de correlación entre el puntaje global de CO (y cada una de sus tres subescalas), con el resto de las variables descritas en el apartado Instrumentos (Método). El CO global correlacionó negativamente con los puntajes globales de confianza en la organización $(r=-.41 ; p<.01)$; compromiso organizacional $(r=-.35 ; p<.01)$; y satisfacción laboral $(r=-.69, p<.01)$. Asimismo, 
se encontraron asociaciones estadísticamente significativas entre las tres facetas del $\mathrm{CO}$ y las subdimensiones que componen cada uno de los constructos explorados, tal como lo muestra la Tabla 4.

\section{Tabla 4}

Medias, desvios típicos y correlaciones bivariadas entre las dimensiones de CO y las subescalas de las variables medidas

\begin{tabular}{ccc}
\hline $\begin{array}{c}\text { Cinismo } \\
\text { organizacional }\end{array}$ & $\begin{array}{c}\text { Confianza } \\
\text { organizacional }\end{array}$ & $\begin{array}{c}\text { Compromiso } \\
\text { organizacional }\end{array}$
\end{tabular}

\begin{tabular}{lcccccccccccccc}
\hline & Media & DT & 1 & 2 & 3 & 4 & 5 & 6 & 7 & 8 & 9 & 10 & 11 & 12 \\
\hline 1 & 2.83 & .68 & -- & & & & & & & & & & & \\
2 & 3.01 & .92 & .54 & -- & & & & & & & & & & \\
3 & 2.46 & .90 & .61 & .66 & -- & & & & & & & & & \\
4 & 3.15 & .89 & -.52 & -.39 & -.36 & -- & & & & & & & & \\
5 & 3.22 & .87 & -.21 & -.19 & -.26 & .44 & - & & & & & & & \\
6 & 3.08 & .99 & -.33 & -.37 & -.36 & .59 & .42 & -- & & & & & & \\
7 & 2.08 & .88 & -.31 & -.21 & -.30 & .17 & .44 & .24 & -- & & & & & \\
8 & 3.64 & .88 & -.49 & -.43 & -.37 & .71 & .38 & .47 & .28 & -- & & & & \\
9 & 3.15 & .98 & -.40 & -.36 & -.39 & .60 & .34 & .49 & .34 & .70 & -- & & & \\
10 & 2.84 & .73 & -.21 & -.26 & -.17 & .45 & .25 & .42 & .45 & .49 & .69 & -- & & \\
11 & 3.07 & .71 & .14 & -.15 & -.11 & .17 & .14 & .23 & .15 & .33 & .37 & .46 & -- & \\
12 & 3.37 & .74 & -.48 & -.41 & -.49 & .71 & .51 & .63 & .31 & .71 & .74 & .55 & .25 & -- \\
\hline
\end{tabular}

Nota: $p<.05$ (apaisada); $p<.01$ (negrita)

Nota: Cinismo organizacional (1: ideas cínicas, 2: conductas cínicas y 3: emociones cínicas); Confianza organizacional (4: promoción del crecimiento, 5: solidez organizacional, 6: reconocimiento financiero, 7: normativas de despido y 8: patrones éticos); Compromiso organizacional (9: compromiso afectivo, 10: compromiso normativo y 11: compromiso calculativo); 12: S.L. (satisfacción laboral).

Como se desprende de la Tabla 4, las tres facetas que integran el CO (Ideas Cínicas, Conductas Cínicas y Emociones Cínicas), se correlacionan negativamente con las cinco dimensiones de la confianza en la organización (Promoción del crecimiento; Solidez organizacional; Reconocimiento financiero; Normativas de despido; Patrones éticos). Se observan, asimismo, vinculaciones negativas entre las facetas afectiva y normativa 
del compromiso organizacional, y las tres subescalas del CO. En lo que respecta al compromiso calculativo, si bien se asocia negativamente a las conductas y emociones cínicas, presenta correlaciones positivas con las ideas cínicas.

\section{Discusión}

Los resultados muestran que a partir de la adaptación realizada se ha logrado una versión parsimoniosa, confiable y válida de la escala para medir CO en trabajadores argentinos. Se trata de una versión integrada por solo 10 ítems, que exploran las diferentes facetas del constructo $\mathrm{CO}$ con un alto nivel de homogeneidad y consistencia interna.

Con respecto a la validez estructural de la escala, los tres factores identificados a través del AFE y confirmados por los AFC, demuestran la naturaleza multidimensional del constructo. La estructura obtenida reproduce la solución trifactorial encontrada tanto por los autores del instrumento (Brandes et al., 1999), como la informada en estudios de validación previos (Bobbio et al., 2006; Karacaoğlu \& İnce, 2012). En este caso, la versión argentina explora los factores rotulados como Ideas, Conductas y Emociones cínicas, respectivamente. El factor Ideas Cínicas incluye tres ítems referidos a las cogniciones sobre la deshonestidad e impericia de la organización; el factor Conductas Cínicas nuclea 4 ítems referidos a comportamientos críticos y sarcásticos, en tanto que el factor Emociones Cínicas agrupa 3 ítems referidos a sentimientos negativas hacia la empresa. Estos tres factores se relacionan significativamente entre sí, lo que indica que se trata de tres facetas de un mismo constructo. Sin embargo, tales interrelaciones no llegan a ser lo suficientemente importantes como para generar problemas de multicolinealidad (Kline, 2011).

La validez convergente de la escala queda demostrada a partir de la fuerza y dirección de las correlaciones obtenidas entre las tres dimensiones del $\mathrm{CO}$ y las restantes variables medidas. En este sentido, las asociaciones negativas entre $\mathrm{CO}$ y la confianza en la organización, no hacen más 
que confirmar que la desconfianza es uno de los pilares sobre los que se asienta el cinismo. Tales asociaciones armonizan también con la evidencia empírica publicada (Chiaburu et al., 2013; Kannan-Narasimhan \& Lawrence, 2012; Kim, Bateman, Gilbreath \& Andersson, 2009), la que señala que los empleados cínicos desarrollan una marcada suspicacia que impide sostener expectativas optimistas tanto sobre las personas (en este caso, líderes, supervisores, directivos organizacionales, etc.), como sobre los resultados de un evento (por ejemplo, el cambio organizacional). Mientras que los empleados confiados consideran que la empresa dejará de lado sus propios intereses a favor del colectivo, los cínicos se muestran convencidos que la organización actuará impulsada por intereses egoístas. La certeza sobre la falta de integridad, los predispone a dudar de cualquier acción que la empresa lleve a cabo y a sospechar que tras las razones explícitas existen motivaciones ocultas. En este sentido, por ejemplo, los cínicos creen que las organizaciones en las que trabajan no brindan oportunidades reales de crecimiento ni tampoco incentivan el desarrollo de sus carreras. Sumado a eso, consideran que su esfuerzo no es reconocido ni valorado económicamente a través del salario que perciben, llegando muchas veces a sentirse "explotados" por sus empleadores. En estas condiciones, es factible que sobrevenga un estado de malestar emocional, así como conductas críticas y despectivas hacia la organización (Salessi \& Omar, 2014).

Las dimensiones de la confianza organizacional que se relacionan más negativamente con el $\mathrm{CO}$ son las referidas a las percepciones de solidez organizacional, de normativas en materia de despido y de los principios éticos de la empresa. En lo que hace a las percepciones de solidez organizacional, es factible presuponer que los cínicos desarrollarán ideas suspicaces respecto a la solvencia y estabilidad económica de la organización; y que frente a una inminente crisis financiera, se mostrarán convencidos que la organización es esencialmente egoísta. Tales creencias podrían retroalimentar emociones como el enojo y la irritación, además de motivar conductas críticas y quejumbrosas, lo que estaría explicando las correlaciones negativas entre esta dimensión de la confianza organizacional y las tres facetas del CO. Las percepciones 
de falta de normativas claras en materia de despido resultan coherentes si se considera que desde la perspectiva del cínico organizacional, un empleado puede ser despedido arbitrariamente porque los directivos tienden a basarse más en juicios personales, que en reglamentaciones legales. El estado de suspicacia e inseguridad que promueven tales ideas conlleva, inevitablemente, a un incremento de la afectividad negativa y a conductas displicentes contra la organización como una forma de lidiar con una situación que se considera injusta y deshonesta. Por último, las correlaciones negativas observadas entre la percepción de patrones éticos y las tres facetas del CO proporcionan nuevo apoyo empírico a los resultados comunicados previamente en la literatura. La teoría clásica sobre CO (Abraham, 2000; Andersson \& Bateman, 1997; Dean et al., 1998), ha subrayado que el cínico tiene la firme convicción que la organización en la que trabaja no es ética; y que los principios de honestidad, justicia y equidad han sido socavados a favor de los intereses personales de la alta gerencia. Por lo que la certeza sobre la forma deshonesta e inescrupulosa con la que los directivos se manejan a la hora de alcanzar sus metas podría suscitar, lógicamente, un estado de enojo, irritación y pesimismo, acentuando la predisposición del cínico a reprochar y cuestionar las prácticas y políticas organizacionales.

En lo que hace a las relaciones entre el compromiso organizacional y el CO, no hay que perder de vista que los trabajadores cínicos tienen inconvenientes para entablar un vínculo afectivo con la organización empleadora. Tal como lo han destacado diversos autores (Rubin, Dierdorff, Bommer \& Baldwin, 2009; Türköz, et al., 2013; Watt \& Piotrowsky, 2008), los cínicos no logran identificarse con la cultura organizacional, ni pueden contribuir proactivamente para que la empresa alcance sus metas y objetivos. En lugar de desarrollar una ligazón emocional con la organización, los cínicos experimentan una amplia gama de sentimientos negativos, que van desde el enojo hasta la desesperanza, tal como lo indican las vinculaciones negativas entre el compromiso afectivo y la emocionalidad cínica. El hallazgo de asociaciones positivas entre las ideas cínicas y el compromiso calculativo, lejos de ser contradictorio se muestra en línea con la naturaleza misma de ambas dimensiones. 
El compromiso calculativo se basa en un análisis especulativo por parte del empleado de los altos costos que le generaría dejar la organización. El trabajador permanece en su puesto porque la razón costo-beneficio le indica que si renuncia, perdería más de lo que ganaría (Omar, 2010). En el caso del cínico, a pesar de su malestar por su condición de miembro de una organización que, desde su punto de vista, es deshonesta e inescrupulosa, concluiría que los perjuicios superarían los beneficios que resultarían de su desvinculación voluntaria. Podría considerar, por ejemplo, que ha hecho grandes sacrificios personales, que su familia se vería seriamente damnificada, que en función de las alternativas del mercado laboral cuenta con escasas posibilidades de encontrar un nuevo trabajo, y similares. En cualquier caso, su decisión de continuar en la organización estaría fundada solo en la necesidad, $y$ no en un deseo genuino de pertenecer y formar parte (lo que en palabras de Jorge Luis Borges se traduciría como "no nos une el amor, sino el espanto..."). Dicha situación queda demostrada por el hecho que, aún frente a niveles elevados de compromiso calculativo, subsisten el malestar emocional y las conductas negativas hacia la organización.

Finalmente, las correlaciones negativas entre los componentes del $\mathrm{CO}$ y la satisfacción laboral proporcionan sustento empírico a conjeturas anticipadas por publicaciones pioneras en la temática (Abraham, 2000; Andersson \& Bateman, 1997). Se ha señalado, por ejemplo, que en un escenario signado por la desconfianza y la falta de deseo de ser parte de la organización en la que se trabaja prevalecerán las respuestas emocionales negativas. En un todo de acuerdo con tales especulaciones teóricas, los resultados obtenidos permiten señalar que los cínicos no experimentan satisfacción laboral, posiblemente por encontrarse en un ambiente en el que no se sienten a gusto y con el que no se vinculan positivamente.

\section{Fortalezas y debilidades de la investigación realizada}

Como todo trabajo empírico, el presente también entraña fortalezas y debilidades. Entre sus limitaciones hay que remarcar la composición de la muestra en estudio, ya que su selección por disponibilidad 
impediría la generalización de los resultados a toda la población de trabajadores argentinos. No obstante, en el intento de reducir el impacto de esta limitación, se procuró que la muestra incluyera empresas e instituciones de los más diferentes rubros y niveles de complejidad organizacional (Podsakoff et al., 2003 ). Otra de las debilidades del estudio podría estar vinculada con el carácter autodescriptivo de los instrumentos empleados para la recolección de datos, aspecto que podría haber generado algunos sesgos derivados de la varianza del método común, así como respuestas contaminadas por la deseabilidad social, considerando que es una temática sensible. En relación a este último aspecto, se tomaron todos los recaudos necesarios para garantizar el carácter anónimo del protocolo de recolección de datos y la confidencialidad de la información evitando, por ejemplo, que jefes y supervisores pudieran tener acceso a la información suministrada por sus subalternos.

Entre las fortalezas, resulta conveniente destacar que este trabajo constituye el primer intento de validar el constructo $\mathrm{CO}$ en un país latinoamericano, siendo el corolario de los esfuerzos que vienen llevándose a cabo en aras de explorar el tema en la región (Salessi, 2011; Salessi \& Omar, 2014). Por lo que representa una contribución genuina para que otros investigadores se interesen por esta problemática y desarrollen estudios empíricos con el recurso de un instrumento con probadas propiedades psicométricas. La validación de la Escala de CO con muestras de sujetos argentinos deja abierta la posibilidad para llevar a cabo estudios de validación en otros países del subcontinente. Esta sería una vía idónea para verificar la validez transcultural del instrumento aquí presentado. Como agenda para futuros estudios, sería valioso realizar estudios comparativos entre empresas de diferentes países a efectos de explorar el impacto de la cultura nacional (operacionalizada a través de los valores culturales de sus miembros) sobre el desarrollo del cinismo, así como entre diferentes empresas de un mismo país a efectos de conocer el impacto de la cultura organizacional (operacionalizada a través de las prácticas de gerenciamiento de recursos humanos) sobre esta perjudicial actitud. 


\section{Implicancias éticas del uso de la Escala de CO}

El CO constituye una peligrosa realidad que se ha infiltrado en las organizaciones contemporáneas, erosionando tanto sus cimientos como el bienestar de sus miembros. Tal es la magnitud que reviste esta problemática que, para algunos autores (Cartwright \& Holmes, 2006), el CO constituye el nuevo paradigma de las relaciones laborales postmodernas. La actual realidad organizacional, signada por el énfasis en la eficiencia y el control ha impactado negativamente en los trabajadores, resultando en una disminución de la confianza y en una renuencia a invertir en la relación laboral. Como resultado, el nivel de desempeño y el compromiso con el trabajo se ven seriamente perjudicados, traduciéndose en última instancia en una pérdida de ingresos para las empresas. Frente a este panorama, es esperable que a las organizaciones les interese identificar rápidamente a los miembros cínicos, con miras a impedir que su eficacia y productividad se vean afectadas. De allí la necesidad de discutir las implicancias éticas consecuentes al uso de un instrumento para medir el CO. En este sentido, el propósito que ha impulsado el presente estudio no ha sido desarrollar una herramienta de control de la cual puedan servirse gerentes y directivos para sancionar e, incluso, desvincular a los empleados que presenten elevados niveles de cinismo. Por el contario, el fin perseguido ha sido dotar a los especialistas organizacionales de una herramienta diagnóstica que pueda proporcionar información para optimizar las condiciones de trabajo, mejorando la efectividad de la empresa y el bienestar de los empleados (Omar, Vaamonde \& Uribe Delgado, 2012).

La escala de CO, lejos de haber sido desarrollada como un dispositivo de vigilancia del comportamiento de los empleados, o un recurso para proceder al castigo u hostigamiento de quienes informen altos niveles de suspicacia; ha sido concebida como un instrumento capaz de aportar información certera en aras de implementar políticas de gestión responsable de los recursos humanos. Se espera que la información proporcionada pueda capitalizarse para el diseño de intervenciones sobre algunos aspectos del funcionamiento de las 
organizaciones, como una forma de contribuir a la reducción de la desconfianza y el pesimismo. Entre tales aspectos, por ejemplo, se ha demostrado que la comunicación efectiva en torno a los cambios (Qian \& Daniels, 2008), el comportamiento ético de las autoridades organizacionales (English \& Chalon, 2011), la transparencia en las compensaciones de la alta gerencia (Welsh, Ganegoda, Arvey, Wiley $\&$ Budd, 2012), la responsabilidad social corporativa (Aqueveque \& Encina, 2010), entre otros, son "antídotos" efectivos para contrarrestar el CO. En otras palabras, las organizaciones que promuevan la confianza en la gestión y se preocupen por crear un clima de integridad y justicia organizacional, se verán beneficiadas con empleados menos cínicos y desconfiados, y más comprometidos y satisfechos.

\section{Referencias}

Abraham, R. (2000). Organizational cynicism: Bases and consequences. Genetic, Social, and General Psychology Monographs, 126, 269-292.

Andersson, L. M. \& Bateman, T. S. (1997). Cynicism in the workplace: Some causes and effects. Journal of Organizational Behavior, 18(5), 449-469.

Aqueveque, C. \& Encina, C. (2010). Corporate behavior, social cynicism, and their effect on individuals' perceptions of the company. Journal of Business Ethics, 91(2), 311-324.

Barton, L. \& Ambrosini, V. (2013). The moderating effect of organizational change cynicism on middle manager strategy commitment. The International Journal of Human Resource Management, 24(4), 721-746.

Bateman, T. S., Sakano, T. \& Fujita, M. (1992). Roger, me, and my attitude: Film propaganda and cynicism toward corporate leadership. Journal of Applied Psychology, 77, 768-771.

Bobbio, A., Manganelli Rattazzi, A. M. \& Spadaro, S. (2006). Il cinismo organizzativo. Un contributo all'adattamento Italiano 
della scala di Brandes, Dharwadkar e Dean (1999). Testing Psicometria Metodologia, 13(1), 5-23.

Brandes, P. \& Das, D. (2006). Locating behavioral cynicism at work: Construct issues and performance implications. In P. L. Perrewé \& D. C. Ganster (Eds.), Employee health, coping and methodologies (pp. 233-266). Philidelphia: Elsevier Science.

Brandes, P., Das, D. \& Hadani, M. (2006). Organizational cynicism: A field examination using global and local social exchange relationships and workplace outcomes. In G. Graen \& J. Graen (Eds.), Sharing Network Leadership (pp. 191-224). Greenwich: Information Age Publishing.

Brandes, P., Dharwadkar, R. \& Dean, J. (1999). Does organizational cynicism matter? Employee and supervisor perspectives on work outcomes. Eastern Academy of Management Best Papers Proceedings, 150-153.

Byrne, B. M. (2010). Structural equation modeling with Amos: Basic concepts, applications, and programming (2 ${ }^{\text {nd }}$ Ed.). New York: Taylor and Francis Group.

Cartwright, S. \& Holmes, N. (2006). The meaning of work: The challenge of regaining employee engagement and reducing cynicism. Human Resource Management Review 16, 199-208.

Chiaburu, D. S., Peng, A. C., Oh, I., Banks, G. C. \& Lomeli, L. C. (2013). Antecedents and consequences of employee organizational cynicism: A meta-analysis. Journal of Vocational Behavior, 83(2), 181-197.

Cook, W. W. \& Medley, D. M. (1954). Proposed hostility and pharisaic-virtue scales for the MMPI. Journal of Applied Psychology, 38, 414-418.

Dean, J. W., Brandes, P. \& Dharwadkar, R. (1998). Organizational cynicism. Academy of Management Review, 23, 341-352.

Didier Pino, N., Martí del Campo, A. \& Valdenegro Ibarra, D. (2012). Manejo del cinismo organizacional: La oportunidad del líder. Revista Iberoamericana de Psicología: Ciencia y Tecnología, 5(2), 7-15. 
English, B. \& Chalon, C. (2011). Strengthening affective organizational commitment: The influence of fairness perceptions of management practices and underlying employee cynicism. The Health Care Manager, 30(1), 29-35.

Evans, W., Goodman, J. \& Davis, W. (2011). The impact of perceived corporate citizenship on organizational cynicism, OCB, and employee deviance. Human Performance, 24(1), 79-97.

Fleming, P. \& Spicer, A. (2003). Working at a cynical distance: Implications for power subjectivity and resistance. Organization, 10(1), 157-179.

Flores, J. M. (2011). Predictores de satisfacción laboral. Un estudio en empresas metal-mecánicas santafesinas. Tesis Inédita de Maestría en Administración de Negocios. Universidad Tecnológica Nacional, Facultad Regional Rosario.

Gkorezis, P., Petridou, E. \& Xanthiakos, P. (2014). Leader positive humor and organizational cynicism: LMX as a mediator. Leadership \& Organization Development Journal, 35(4), 305-315.

Grama, B. (2013). Cinism în schimbările organizaţionale. Management Intercultural, 29(3), 137-142.

Hair, J. E., Black, W. C., Babin, B. J., Anderson, R. E. \& Tatham R. L. (2006). Multivariate Data Analysis (6 ${ }^{\text {th }}$ Ed.). Upper Saddle River: Pearson-Prentice Hall.

Hasselmann, M. H. \& Reichenheim, M. E. (2003). Cross-cultural adaptation of the Portuguese version of the Conflict Tactics Scales Form R (CTS-1) used to assess marital violence: Semantic and measurement equivalence. Cadernos de Saúde Pública, 19(4), 1083-1093.

Herdman, M., Fox-Rushby, J. \& Badía, X. (1998). A Model of equivalence in the cultural adaptation of HRQOL instruments: The universalist approach. Quality of Life Research, 7(4), 323-335.

Kan, M. A. (2014). Organizational cynicism and employee turnover intention: Evidence from banking sector in Pakistan. Pakistan Journal of Commerce \& Social Sciences, 8(1), 30- 41. 
Kannan-Narasimhan, R. \& Lawrence, B. (2012). Behavioral integrity: How leader referents and trust matter to workplace outcomes. Journal of Business Ethics, 111(2), 165-178.

Kanter, D. L. \& Mirvis, P. H. (1989). The cynical Americans: Living and working in an age of discontent and disillusionment. San Francisco: Jossey-Bass.

Karacaoğlu, K. \& Ince, G. F. (2012). The effects of positive organizational behavior on organizational cynicism: A case study Kayseri's manufacturing industry. Journal of Faculty of Economics \& Administrative Sciences, 18(1), 181-202.

Kim, T., Bateman, T., Gilbreath, L. \& Andersson, L. (2009). Top management credibility and employee cynicism: A comprehensive model. Human Relations, 62(10), 1435-1458.

Kline, R. B. (2011). Principles and practice of structural equation modeling ( ${ }^{\text {ed }}$ Ed.). New York: Guilford.

Macdonald, S. \& MacIntyre, P. (1997). The generic job satisfaction scale. Employee Assistance Quarterly, 13, 1-16.

Martínez Lugo, M., Aponte, R., Quintero, M., Lindenmann, M., Gómez, K., Vaello, G. et al. (2005, 4 de noviembre). El sindrome de quemarse por el trabajo y el cinismo organizacional: Estudio exploratorio. Ponencia presentada en la Quincuagésimo Segunda Convención Anual de la Asociación de Psicología de Puerto Rico. San Juan, Puerto Rico.

Meyer, J. P. \& Allen, N. J. (1991). A three component conceptualization of organizational commitment, Human Resource Management Review, 1, 61-98.

Neves, P. (2012). Organizational cynicism: Spillover effects on supervisor-subordinate relationships and performance. The Leadership Quarterly 23, 965-976.

Nunnally, J. C. \& Bernstein, I. H. (1994). Psychometric theory ( $3^{\text {rd }}$ Ed.). New York: McGraw-Hil.

Oliveira, A. F. \& Tamayo, A. (2008). Confianza do empregado na organização. Em M. M. Siqueira \& A. Tamayo (Eds.), Medidas do comportamento organizacional (pp. 165-280). Porto Alegre: Artmed. 
Omar, A. (2006). La cultura organizacional de empresas e instituciones argentinas a través de las prácticas, actitudes y comportamientos de sus miembros. Rosario: CONICET.

Omar, A. (2010). Las organizaciones positivas. En A. Castro Solano (Ed.), Fundamentos de Psicología Positiva (pp. 137-182). Buenos Aires: Paidós.

Omar, A. (2011). Liderazgo transformador y satisfacción laboral: El rol de la confianza en el supervisor. Liberabit, 17(2), 129-137.

Omar, A., Vaamonde, J. D. \& Uribe Delgado, H. (2012). Comportamientos contraproducentes en el trabajo: Diseño y validación de una escala Diversitas: Perspectivas en Psicología, 8(2), 249-265.

Podsakoff, P. M., MacKenzie, S. B., Lee, J. Y. \& Podsakoff, N. P. (2003). Common method biases in behavioral research: A critical review of the literature and recommended remedies. Journal of Applied Psychology, 88(5), 879-903.

Qian, Y. \& Daniels, T. D. (2008). A communication model of employee cynicism toward organizational change. Corporate Communications: An International Journal, 13, 319-332.

Reichers, A. E., Wanous, J. P. \& Austin, J. T. (1997). Understanding and managing cynicism about organizational change. Academy of Management Executive, 11, 48-59.

Rubin, R., Dierdorff, E., Bommer, W. \& Baldwin, T. (2009). Do leaders reap what they sow? Leader and employee outcomes of leader organizational cynicism about change. The Leadership Quarterly, 20(5), 680-688.

Salessi, S. (2011). Cinismo Organizacional: Una revisión de la literatura y algunas consideraciones conceptuales. Revista Interamericana de Psicología Ocupacional, 30(1), 88-105.

Salessi, S. \& Omar, A. (2014). Cinismo Organizacional: Consecuencias sobre la salud mental de los trabajadores. Psicología y Salud, 24(2), 269-277.

Sarnoff, I. (1960). Reaction formation and cynicism. Journal of Personality, 28(1), 129-143. 
Schumacker, R.E. \& Lomax, R.G. (2004). A beginner's guide to structural equation modeling $\left(2^{\text {nd }} \mathrm{Ed}\right.$.). Mahwah: Lawrence Erlbaum Associates.

Stanley, D. J., Meyer, J. P. \& Topolnytsky, L. (2005). Employee cynicism and resistance to organizational change. Journal of Business \& Psychology, 19(4), 429-459.

Tabachnick, B. G. \& Fidell, L. S. (2007). Using Multivariate Statistics ( $5^{\text {th }}$ Ed.). Boston: Allyn \& Bacon.

Türköz, T., Polat M. \& Coțar, S. (2013). The role of employees' organizational trust and cynicism perceptions on organizational commitment. Journal of Management \& Economics, 20(2), 285302.

Wanous, J. P., Reichers, A. E. \& Austin, J. T. (2000). Cynicism about organizational change: Measurement, antecedents, and correlates. Group \& Organization Management, 25, 132-153.

Watt, J. D. \& Piotrowski, C. (2008). Organizational change cynicism: A review of the literature and intervention strategies. Organization Development Journal, 26(3), 23-31.

Welsh, E. T., Ganegoda, D. B., Arvey, R. D., Wiley, J. W. \& Budd, J. W. (2012). Is there fire? Executive compensation and employee attitudes. Personnel Review, 41(3), 260-282.

Recibido: 30 de junio, 2014 Aceptado: 29 de julio, 2014 

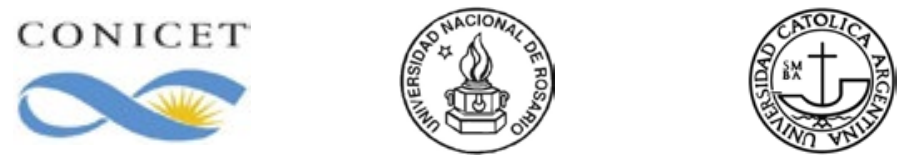

Consejo Nacional de Investigaciones Científicas y Técnicas (CONICET)

Universidad Nacional de Rosario (UNR)

Pontificia Universidad Católica Argentina (UCA)

\section{CONSENTIMIENTO INFORMADO}

Estimada/o participante:

Estamos realizando una investigación avalada por el CONICET, la Universidad Nacional de Rosario y la Pontificia Universidad Católica Argentina, con el propósito conocer mejor la manera habitual de actuar, pensar y sentir de los trabajadores argentinos.

Su colaboración en el estudio consistirá en completar el cuestionario adjunto, actividad que le tomará alrededor de 20 minutos. Usted tiene total libertad de participar o no en esta recolección de datos, así como también de abandonar su colaboración en cualquier momento del proceso.

La información recopilada mediante su participación será estricta y exclusivamente para nuestro trabajo de investigación, y solo nosotros tendremos acceso a la misma. Asimismo, la información que proporcione no estará identificada con su nombre, para resguardar el anonimato y la confidencialidad de sus respuestas.

Su participación en esta investigación no implica ningún beneficio monetario o de otra clase. No obstante, su colaboración es muy importante para el éxito de este estudio y de futuros estudios relacionados con el tema de investigación.

En caso de tener alguna consulta o sugerencia, no dude en comunicarse con nosotros:
Dra. Alicia Omar
(CONICET-UNR)
Lic. Solana Salessi
(CONICET-UCA)

Fac. Humanidades y Artes UNR

Entre Ríos 758 - Rosario

Tel. (0341) 4802673

agomar@yahoo.com.ar
Fac. de Derecho y Cs. Sociales UCA

Av. Pellegrini 3314 - Rosario

Tel. (0341) 436-8000

solanasalessi@gmail.com

Expreso voluntaria y conscientemente mi consentimiento de participar en el estudio. No deseo participar del estudio.

Firma: 